\title{
Determinants of Low Birth Weight Deliveries: an Unmatched Case-control Study in Five Referral Hospitals in Western Area Urban District, Sierra Leone
}

\section{David Kabba Kargbo}

Field Epidemiology Training Program, Sierra Leone

Kofi Nyarko

Field Epidemiology Training Program, Sierra Leone

\section{Samuel Sackey}

Field Epidemiology and Laboratory Training Programme, Ghana Biometry: National Institute on Aging Intramural Research Program

\section{Adolphina Addo-Lartey}

Department of Epidemiology and Disease Control, School of Public Health, University of Ghana

\section{Ernest Kenu}

Field Epidemiology and Laboratory Training Programme, Ghana

Francis Anto ( $\square$ fanto@ug.edu.gh )

School of Public Health, University of Ghana https://orcid.org/0000-0002-1531-5481

\section{Research}

Keywords: Low birth weight, Case-control, Referral Hospitals, Western Area Urban, Sierra Leone

Posted Date: January 8th, 2021

DOl: https://doi.org/10.21203/rs.3.rs-139964/v1

License: (c) (i) This work is licensed under a Creative Commons Attribution 4.0 International License. Read Full License 


\section{Abstract}

Background: Each year, about 20 million deliveries are Low Birth Weight (LBW) with 96.5\% occurring in developing countries. The prevalence of LBW in Sierra Leone is about 7\% while the Western Area Urban (WAU) district reports $17.5 \%$. This study sought to determine factors associated with LBW deliveries in five referral hospitals in the WAU district, Sierra Leone.

Methods: A hospital-based unmatched 1:2 case-control study was conducted among mothers who delivered live singleton babies from November, 2019 to February, 2020 in five referral facilities. Antenatal care cards of mothers were reviewed and a pre-tested questionnaire also administered to them. The association between maternal socio-demographic, socio-economic, obstetric and lifestyle factors and LBW was assessed using bivariable and multivariable logistic regression analyses.

Results: A total of 438 mothers (146 cases and 292 controls), mean age: $24.2( \pm 5.8)$ and $26.1( \pm 5.5)$ years for cases and controls respectively participated in the study. Among mothers, $36.9 \%(162 / 438)$ had no formal education, 25.8\% (113/438) were unemployed and 29.7\% (130/438) were unmarried.

Multivariable analysis revealed that being unemployed ( $\mathrm{AoR}=2.52,95 \% \mathrm{Cl} 1.16-5.49, \mathrm{p}=0.020)$, having anaemia during pregnancy $(\mathrm{AoR}=3.88,95 \% \mathrm{Cl} 1.90-7.90, \mathrm{p}<0.001)$, having less than two years interpregnancy interval ( $\mathrm{AoR}=2.53,95 \% \mathrm{Cl} 1.11-5.73, \mathrm{p}=0.026)$, and smoking cigarettes during pregnancy (AoR $=4.36,95 \% \mathrm{Cl} 1.94-9.80, \mathrm{p}<0.001)$ were significantly associated with having LBW babies.

Conclusion: Factors associated with LBW identified were unemployment, anaemia during pregnancy, $<2$ years inter-pregnancy interval and cigarette smoking during pregnancy. Health care providers should screen and sensitize mothers on the risk factors of LBW during antenatal sessions.

\section{Background}

Low birth weight (LBW) is of major public health concern especially in low and middle-income countries [1]. It contributes significantly to the burden of childhood diseases and increases mortality and disability in neonates and infants [2]. LBW is the weight of a newborn measuring less than $2.5 \mathrm{~kg}$ and is measured immediately after birth [3].

Newborns with LBW are at greater risk of neonatal mortality compared to normal weight babies [4]. Management of LBW deliveries increases health care costs due to long periods of hospitalization. It is estimated that care for extremely LBW babies is about six times more expensive compared to normal weight babies [5]. Evidence also exists that LBW increases the likelihood of developing noncommunicable diseases like diabetes and cardiovascular diseases in adulthood [5, 6]. LBW has short term consequences, including respiratory distress, infections and hydrocephalus as well as long-term consequences, including coronary heart disease, diabetes, hypertension, and impaired cognitive function [7]. 
Globally, 15 to $20 \%$ of total births are LBW representing about 20 million births a year [8]. About $96.5 \%$ of LBW deliveries in 2019 occurred in developing countries; accounting for $60-80 \%$ of neonatal deaths [4]. The prevalence of LBW varies from region to region and within countries; with Asia accounting for $28 \%$, Africa $13 \%$ and Latin America 9\% [8].

In Sierra Leone, the pattern of LBW deliveries is unstable. The 2008 and 2013 Sierra Leone Demographic and Health Survey (SLDHS) showed that nationwide, LBW deliveries decreased from $11.0 \%$ in 2008 to $7.1 \%$ in 2013 . However, it rather increased in the Western Area Urban district from 9.5-17.5\% over the same period $[9,10]$.

Several factors including early initiation of labour, mother's lifestyle (e.g. smoking and drinking alcohol), multiple pregnancies, maternal age $<15$ years or $>35$ years, poverty, infections and chronic illnesses including diabetes and hypertension have been reported to contribute to the delivery of LBW babies [6]. The purpose of the current study was to identify factors contributing to LBW in Sierra Leone to allow for the development of sustainable preventive measures in order to reduce the cost to the health sector. This study, therefore, assessed the socio-demographic, obstetric, maternal, and lifestyle factors associated with LBW babies delivered in five referral hospitals in WAU, Sierra Leone.

\section{Methods}

\section{Study design}

A hospital-based unmatched case-control study was undertaken in five referral hospitals in the Western Area Urban district of Sierra Leone from November, 2019 to February, 2020 among mothers who delivered singleton live babies. For each mother who delivered a LBW baby, two mothers who subsequently delivered normal weight babies in the same hospital were selected as controls.

\section{Study setting}

The study was conducted in five-referral hospitals (Government and Non-governmental organization (NGO) supported) offering free maternal services: Princess Christian Maternity Hospital (PCMH), Lumley Government Hospital, King Harman Road Government Hospital, 34 Military hospital, and Aberdeen Women's Centre (NGO). All these facilities were located within the capital city, Freetown. In 2019, the WAU district had a projected population of 1.2 million. The district is divided into 20 zones and has five referral hospitals and 71 peripheral health units (PHUs). The district recorded 6,112 deliveries in health facilities in 2019.

\section{Study variables}

The independent variables included socio-demographic factors (age of mother, educational level, ethnicity, marital status and religion), socio-economic factors, (family income level and occupation), obstetric factors (maternal BMI, weight, height, parity, gravidity, abortion, ANC visits, gestational age, birth interval and anaemia during pregnancy), maternal health and lifestyle factors (diseases like hypertension, 
diabetes, heart disease, HIV, syphilis and malaria, alcohol intake, smoking and use of herbal medication). The dependent variable was low birth weight.

\section{Study participants and eligibility criteria}

Mothers who delivered live singleton babies in the five referral hospitals during the study period were eligible to participate in the study. The weight of every newborn was measured within an hour of birth. Mothers with unknown last normal menstrual period, caesarean section birth, congenital deliveries, stillbirths, seriously ill, and mothers with singletons who weighed more than $4 \mathrm{~kg}$ were excluded from the study.

Cases definition: Mothers who delivered babies weighing less than $2.5 \mathrm{~kg}$ and consented to take part in the study were enrolled as cases.

Control definition: Mothers with newborns weighing $2.5 \mathrm{~kg}$ to $4 \mathrm{~kg}$ were enrolled as controls.

Sample size determination

An online OpenEpi software, version three for unmatched case-control studies was used to estimate the required sample size. A minimum detectable odds ratio of two and a percentage control group (birth spacing less than two years) exposed (67.6\%) from a study conducted in Ethiopia was used [11]. A case to control ratio of $1: 2$ with a $95 \%$ confidence level and $80 \%$ power was used. The required sample size estimated was 438 (146 cases and 292 controls).

\section{Sampling procedure}

A total of 251 singleton live LBW babies were delivered in the five hospitals from November, 2018 to February, 2019. PCMH had 138 records of singleton live LBW deliveries, King Harman Road Government Hospital, three, Lumley Government Hospital, 15, 34 Military hospital, eight and Aberdeen Women's Centre, 87. The sample size was distributed proportionately among the five facilities based on the November, 2018 to February, 2019 singleton LBW deliveries per facility. Mothers were enrolled sequentially as they delivered until the required sample size for the facility was attained. Each mother who gave birth to a singleton live baby weighing $<2.5 \mathrm{~kg}$ was enrolled as a case and two mothers who subsequently delivered singleton live babies weighing $2.5 \mathrm{~kg}$ to $4.0 \mathrm{~kg}$ were also enrolled as controls. In a situation where a case or control declined consent to be part of the study, the next eligible mother was selected as a replacement.

\section{Data collection tools and procedure}

A questionnaire was developed specifically for this study based on the objectives of the study and used for data collection from mothers who met the inclusion criteria of the study. The questionnaire was in three sections: socio-demographic and socio-economic, obstetric, and maternal health and lifestyle factors. The ANC cards of the mothers were reviewed and data on obstetric and maternal health factors extracted. Fifteen trained research assistants (three per hospital and one per shift) collected the data through face to face interviews using the common language used in the study area, 'Creole'. Midwives 
took the weights of newborn babies within one hour after delivery using calibrated Seca weighing scales. The research assistants administered the questionnaires to mothers within 24 hours post-delivery in a separate office within the ward to maintain privacy. Data were collected simultaneously in the five hospitals until the required sample size was obtained.

\section{Quality control}

The questionnaire was developed specifically for this study based on the objectives of the study, known variables from literature and the research experience of the authors. The questionnaire was pre-tested in three Government and NGO supported hospitals in the WAU district with similar service characteristics using $2 \%$ (three cases and six controls) of the sample size (cases). Findings of the pilot study were used to finalize the questionnaire. The data collected were checked daily for completeness and accuracy by a supervisor. The data were cleaned and entered in SPSS version 22 software and password protected.

\section{Data processing and analysis}

Statistical analysis was done using Stata 15.0 (Stata Corp, College Station, TX USA). A histogram-normal curve was used to check the normality of continuous variables. Descriptive analysis i.e. summary statistics, mean (SD) and proportions were estimated, and inferential analysis using chi-square and logistic regression (bivariable and multivariable) to determine association between LBW and the independent variables. A stepwise backward elimination method with a restricted alpha level of $0.1(10 \%)$ was conducted and the post estimation command ("testparm i.variablename") used to determine variables that met the criteria for the multivariable logistic model. Odds ratio (OR) with $95 \% \mathrm{Cl}$ was computed and statistical significance was determined at $p$-value $<0.05$.

\section{Ethical consideration}

Ethical clearance was obtained from the Sierra Leone Ethics and Scientific Review Committee (SLESRC). Permission was also obtained from the Chief Medical Officer, Ministry of Health and Sanitation (MoHS), and the hospital administration. The possible risk of the study (time) was explained to the mothers and their worries addressed. Written informed consent was obtained from each mother or her parent or guardian (mothers less than 18 years old) before data collection. The information collected was treated as confidential and codes were used to identify participants and not names.

\section{Results}

\section{Socio-demographic characteristics of the mothers}

A total of 438 mothers (146 cases and 292 controls) were enrolled into the study from the five-referral hospitals. They were aged 14-39 years, with mean age of 24.2 (SD: 5.8 ) years for cases and 26.1 (SD:

5.5) years for controls. One hundred and sixty-two of the mothers (36.9\%) had no formal education, $25.8 \%$ (113) were unemployed and 29.7\% (130) were not married (Table 1). 
Table 1

Socio-demographic characteristics of the mothers, WAU, 2020

\begin{tabular}{|c|c|c|c|}
\hline Characteristics & Cases (\%) & Controls (\%) & Total (\%) \\
\hline & $n=146$ & $\mathrm{n}=292$ & 438 \\
\hline \multicolumn{4}{|l|}{ Mother's age (years) } \\
\hline$<20$ & $36(24.6)$ & $30(10.3)$ & $66(15.1)$ \\
\hline $20-29$ & $82(56.2)$ & $190(65.1)$ & $272(62.1)$ \\
\hline$\geq 30$ & $28(19.2)$ & $72(24.6)$ & $100(22.8)$ \\
\hline \multicolumn{4}{|l|}{ Education } \\
\hline No formal education & $64(43.8)$ & $98(33.6)$ & $162(36.9)$ \\
\hline Primary & $23(15.8)$ & $40(13.7)$ & $63(14.4)$ \\
\hline Secondary & $42(28.8)$ & $113(38.7)$ & $155(35.4)$ \\
\hline Tertiary & $17(11.6)$ & $41(14.0)$ & $58(13.2)$ \\
\hline \multicolumn{4}{|l|}{ Employment status } \\
\hline Unemployed & $63(43.1)$ & $50(17.1)$ & $113(25.8)$ \\
\hline Student & $20(13.7)$ & $39(13.4)$ & $59(13.5)$ \\
\hline Employed & $63(43.2)$ & $203(69.5)$ & $266(60.7)$ \\
\hline \multicolumn{4}{|l|}{ Mother/household monthly income } \\
\hline$<$ Le 500,000 & $121(82.9)$ & $226(77.4)$ & $347(79.2)$ \\
\hline$\geq$ Le 500,000 & $25(17.1)$ & $66(22.6)$ & $91(20.8)$ \\
\hline \multicolumn{4}{|l|}{ Marital status } \\
\hline Single & $55(37.7)$ & $75(25.7)$ & $130(29.7)$ \\
\hline Cohabiting/married husband unemployed & $15(10.3)$ & $15(5.1)$ & $30(6.8)$ \\
\hline Cohabiting/married husband employed & $76(52.0)$ & $202(69.2)$ & $278(63.5)$ \\
\hline \multicolumn{4}{|l|}{ Religious affiliation } \\
\hline Christian & $60(41.1)$ & $110(37.7)$ & $170(38.8)$ \\
\hline Muslim & $86(58.9)$ & $182(62.3)$ & $268(61.2)$ \\
\hline
\end{tabular}

Bivariable logistic regression analysis of the socio-demographic characteristics of the mothers revealed statistical differences between cases and controls in terms of age (CoR $=3.08,95 \% \mathrm{Cl} 1.60-5.92, p=$ 
0.001), employment ( $\mathrm{CoR}=4.06,95 \% \mathrm{Cl} 2.54-6.47, \mathrm{p}<0.001)$, and marital status ( $\mathrm{CoR}=1.94,95 \% \mathrm{Cl}$ $1.25-3.01, \mathrm{p}=0.003)($ Table 2$)$. 
Table 2

Comparison of socio-demographic and socio-economic characteristics of mothers of cases and controls, WAU, 2020

\begin{tabular}{|c|c|c|c|c|}
\hline Characteristics & $\begin{array}{l}\text { Cases }(n= \\
146)\end{array}$ & $\begin{array}{l}\text { Controls }(n= \\
292)\end{array}$ & $\begin{array}{l}\text { Crude OR } \\
(95 \% \mathrm{Cl})\end{array}$ & $\begin{array}{l}\mathrm{p}- \\
\text { value }\end{array}$ \\
\hline \multicolumn{5}{|l|}{ Mother's age (years) } \\
\hline$<20$ & 36 & 30 & $\begin{array}{l}3.08(1.60- \\
5.92)\end{array}$ & 0.001 \\
\hline $20-29$ & 82 & 190 & $\begin{array}{l}1.10(0.66- \\
1.84)\end{array}$ & 0.688 \\
\hline$\geq 30$ & 28 & 72 & Ref & \\
\hline \multicolumn{5}{|l|}{ Mother's highest education } \\
\hline No formal education & 64 & 98 & $\begin{array}{l}1.57(0.82- \\
3.00)\end{array}$ & 0.169 \\
\hline Primary & 23 & 40 & $\begin{array}{l}1.38(0.64- \\
2.97)\end{array}$ & 0.401 \\
\hline Secondary/Tec-Voc & 42 & 113 & $\begin{array}{l}0.89(0.45- \\
1.74)\end{array}$ & 0.748 \\
\hline Tertiary & 17 & 41 & Ref & \\
\hline \multicolumn{5}{|l|}{ Mother's employment } \\
\hline Unemployed & 63 & 50 & $\begin{array}{l}4.06(2.54- \\
6.47)\end{array}$ & $<.001$ \\
\hline Student & 20 & 39 & $\begin{array}{l}1.65(0.89- \\
3.03)\end{array}$ & 0.106 \\
\hline Employed/self-employed & 63 & 203 & Ref & \\
\hline \multicolumn{5}{|c|}{ Mother/household monthly income } \\
\hline$<$ Le 500,000 & 121 & 226 & $\begin{array}{l}1.41(0.83- \\
2.46)\end{array}$ & 0.182 \\
\hline$\geq$ Le 500,000 & 25 & 66 & Ref & \\
\hline \multicolumn{5}{|l|}{ Marital status } \\
\hline Single & 55 & 75 & $\begin{array}{l}1.94(1.25- \\
3.01)\end{array}$ & 0.003 \\
\hline $\begin{array}{l}\text { Cohabiting/married husband } \\
\text { unemployed }\end{array}$ & 15 & 15 & $\begin{array}{l}2.65(1.23- \\
5.69)\end{array}$ & 0.012 \\
\hline
\end{tabular}

Crude odd ratio (OR), $\mathrm{Cl}$ (confidence interval) 


\begin{tabular}{|lllll|}
\hline Characteristics & $\begin{array}{l}\text { Cases }(n= \\
\text { 146) }\end{array}$ & $\begin{array}{l}\text { Controls }(n= \\
\text { 292) }\end{array}$ & $\begin{array}{l}\text { Crude OR } \\
(95 \% \text { Cl })\end{array}$ & $\begin{array}{l}\text { p- } \\
\text { value }\end{array}$ \\
\hline $\begin{array}{l}\text { Cohabiting/married husband } \\
\text { employed }\end{array}$ & 76 & 202 & Ref & \\
\hline $\begin{array}{l}\text { Religious affiliation } \\
\text { Christian }\end{array}$ & 60 & 110 & $\begin{array}{l}1.15(0.75- \\
1.76)\end{array}$ & 0.488 \\
\hline Muslim & 86 & 182 & Ref & \\
\hline Crude odd ratio (OR), Cl (confidence interval) & & & \\
\hline
\end{tabular}

\section{Obstetric characteristics of the mothers}

Table 3 displays the obstetric factors found to be significantly associated with delivery of low birth weight babies after bivariable analysis. These factors included, maternal height (CoR $=2.19,95 \% \mathrm{Cl}$ $1.34-3.55, p=0.001)$, gravidity (CoR $=1.81,95 \% \mathrm{Cl} 1.18-2.76, p=0.003)$, number of ANC visits made before delivery $(\mathrm{CoR}=2.69,95 \% \mathrm{Cl} 1.70-4.26, \mathrm{p}<0.001)$, and gestational age at delivery $(\mathrm{CoR}=12.58$, $95 \% \mathrm{Cl} 1.66-94.84, \mathrm{p}=0.014)$. Other factors are anaemia during pregnancy $(\mathrm{CoR}=4.56,95 \% \mathrm{Cl} 2.90-$ $7.16, \mathrm{p}<0.001)$, and birth spacing ( $\mathrm{CoR}=2.26,95 \% \mathrm{Cl} 1.15-4.39, \mathrm{p}=0.008)$.

\section{Maternal health and lifestyle factors}

Bivariable analysis showed statistically significant differences between cases and controls regarding maternal health factors: hypertension status ( $\mathrm{CoR}=4.47,95 \% \mathrm{Cl} 2.57-7.83, \mathrm{p}=<0.001$ ), HIV status (CoR $=3.31,95 \% \mathrm{Cl} 1.14-10.29, \mathrm{p}=0.015)$, syphilis infection $(\mathrm{CoR}=3.87,95 \% \mathrm{Cl} 2.01-7.55, \mathrm{p}<0.001)$, malaria during pregnancy $(\mathrm{CoR}=2.99,95 \% \mathrm{Cl} 1.91-4.73, \mathrm{p}<0.001)$, duration of iron and folic acid use (CoR = $3.55,95 \% \mathrm{Cl} 1.92-6.60, \mathrm{p}<0.001)$. The lifestyle factors associated with delivery of LBW babies were: smoking or living with a partner who smokes ( $\mathrm{CoR}=5.01,95 \% \mathrm{Cl} 2.92-8.67, \mathrm{p}<0.001)$ and $(\mathrm{CoR}=2.31$, $95 \% \mathrm{Cl} 1.49-3.56, \mathrm{p}<0.001)$ respectively, and taking herbal medicines during pregnancy ( $\mathrm{CoR}=3.32,95 \%$ Cl 2.14-5.15, $p<0.001$ ) (Table 4). 
Table 3

Obstetric factors associated with delivery of low birth weight babies, WAU, 2020

\begin{tabular}{|c|c|c|c|c|}
\hline Characteristics & $\begin{array}{l}\text { Cases }(n= \\
146)\end{array}$ & $\begin{array}{l}\text { Controls }(n= \\
292)\end{array}$ & Crude OR (95\% Cl) & $p$-value \\
\hline \multicolumn{5}{|l|}{ Mother's weight (kg) } \\
\hline$<50$ & 4 & 9 & $0.88(0.19-3.24)$ & 0.842 \\
\hline$\geq 50$ & 142 & 283 & Ref & \\
\hline \multicolumn{5}{|l|}{ Mother's height (m) } \\
\hline$<1.5$ (short) & 47 & 52 & $2.19(1.34-3.55)$ & 0.001 \\
\hline$\geq 1.5$ (normal) & 99 & 240 & Ref & \\
\hline \multicolumn{5}{|l|}{ Mother's BMI $\left(\mathrm{kg} / \mathrm{m}^{2}\right)$} \\
\hline$<18.5$ (underweight) & 4 & 9 & $1.26(0.35-4.45)$ & 0.715 \\
\hline 18.5-24.9 (normal) & 35 & 48 & $2.07(1.11-3.87$ & 0.022 \\
\hline 25-29.9 (overweight) & 81 & 161 & $1.43(0.85-2.40)$ & 0.176 \\
\hline$\geq 30$ (obese) & 26 & 74 & Ref & \\
\hline \multicolumn{5}{|l|}{ Parity } \\
\hline Primiparous & 83 & 148 & $1.28(0.84-1.95)$ & 0.223 \\
\hline Multiparous & 63 & 144 & Ref & \\
\hline \multicolumn{5}{|l|}{ Gravidity } \\
\hline Primigravida & 80 & 117 & $1.81(1.18-2.76)$ & 0.003 \\
\hline Multigravida & 66 & 175 & Ref & \\
\hline \multicolumn{5}{|l|}{ ANC Visits } \\
\hline$<4$ times & 60 & 60 & $2.69(1.70-4.26)$ & $<0.001$ \\
\hline$\geq 4$ times & 86 & 232 & Ref & \\
\hline \multicolumn{5}{|l|}{ Gestational age (weeks) } \\
\hline Preterm $(<37)$ & 134 & 213 & $\begin{array}{l}12.58(1.66- \\
94.84)\end{array}$ & 0.014 \\
\hline Term (37-40) & 11 & 59 & $3.72(0.45-30.72)$ & 0.221 \\
\hline Post term $(>40)$ & 1 & 20 & Ref & \\
\hline \multicolumn{5}{|l|}{ Anaemia during pregnancy } \\
\hline Anaemic $(\mathrm{Hb}<11.0 \mathrm{~g} / \mathrm{dl})$ & 82 & 64 & $4.56(2.90-7.16)$ & $<0.001$ \\
\hline
\end{tabular}




\begin{tabular}{|lllll|}
\hline Characteristics & $\begin{array}{l}\text { Cases }(n= \\
\text { 146) }\end{array}$ & $\begin{array}{l}\text { Controls }(n= \\
\text { 292 })\end{array}$ & Crude OR (95\% Cl) & $p$-value \\
\hline $\begin{array}{l}\text { Not anaemic }(\mathrm{Hb} \geq \\
11.0 \mathrm{~g} / \mathrm{dl})\end{array}$ & 64 & 228 & Ref & \\
\hline Birth spacing & & & & \\
\hline$<2$ years & 24 & 29 & $2.26(1.15-4.39)$ & 0.008 \\
\hline$\geq 2$ years & 58 & 159 & Ref & \\
\hline Previous abortion & & & & 0.398 \\
\hline Ever had & 42 & 73 & $1.21(0.75-1.93)$ & \\
\hline Never had & 104 & 219 & Ref & \\
\hline
\end{tabular}


Table 4

Maternal health and lifestyle factors associated with delivery of LBW babies, WAU, 2020

\begin{tabular}{|c|c|c|c|c|}
\hline Determinants & $\begin{array}{l}\text { Cases }(n= \\
146)\end{array}$ & $\begin{array}{l}\text { Controls }(n= \\
292)\end{array}$ & $\begin{array}{l}\text { Crude OR } \\
(95 \% \mathrm{Cl})\end{array}$ & $\begin{array}{l}P \\
\text { value }\end{array}$ \\
\hline \multicolumn{5}{|l|}{ Diabetes } \\
\hline Diabetic & 3 & 18 & $0.31(0.05-1.12)$ & 0.057 \\
\hline Not Diabetic & 143 & 274 & Ref & \\
\hline \multicolumn{5}{|l|}{ Hypertension } \\
\hline Hypertensive & 47 & 28 & $4.47(2.57-7.83)$ & $<0.001$ \\
\hline Not hypertensive & 99 & 264 & Ref & \\
\hline \multicolumn{5}{|l|}{ Heart disease } \\
\hline Heart disease & 3 & 5 & $1.20(0.18-6.28)$ & 0.800 \\
\hline No Heart disease & 143 & 287 & Ref & \\
\hline \multicolumn{5}{|l|}{ HIV } \\
\hline Positive & 11 & 7 & $\begin{array}{l}3.31(1.14- \\
10.29)\end{array}$ & 0.010 \\
\hline Negative & 135 & 285 & Ref & \\
\hline \multicolumn{5}{|l|}{ Syphilis } \\
\hline Positive & 31 & 19 & $3.87(2.01-7.55)$ & $<0.001$ \\
\hline Negative & 115 & 273 & Ref & \\
\hline \multicolumn{5}{|l|}{ Malaria } \\
\hline Positive & 106 & 137 & $2.99(1.91-4.73)$ & $<0.001$ \\
\hline Negative & 40 & 155 & Ref & \\
\hline \multicolumn{5}{|c|}{ Iron \& folic acid used } \\
\hline$<3$ months & 34 & 23 & $3.55(1.92-6.60)$ & $<0.001$ \\
\hline$\geq 3$ months & 112 & 269 & Ref & \\
\hline \multicolumn{5}{|l|}{ Alcohol use } \\
\hline Takes alcohol & 17 & 23 & $1.54(0.74-3.13)$ & 0.197 \\
\hline Does not take & 129 & 269 & Ref & \\
\hline Smoking & & & & \\
\hline
\end{tabular}




\begin{tabular}{|lllll|}
\hline Determinants & $\begin{array}{l}\text { Cases }(\mathbf{n}= \\
\mathbf{1 4 6})\end{array}$ & $\begin{array}{l}\text { Controls }(\mathbf{n}= \\
\mathbf{2 9 2})\end{array}$ & $\begin{array}{l}\text { Crude OR } \\
(95 \% \mathrm{Cl})\end{array}$ & $\begin{array}{l}\boldsymbol{P} \\
\text { value }\end{array}$ \\
\hline Smokes & 52 & 29 & $5.01(2.92-8.67)$ & $<0.001$ \\
\hline Do not smoke & 94 & 263 & Ref & \\
\hline $\begin{array}{l}\text { Living with partner that } \\
\text { smoked }\end{array}$ & & & & \\
\hline Partner smokes & 68 & 80 & $2.31(1.49-3.56)$ & $<0.001$ \\
\hline Partner does not smoke & 78 & 212 & Ref & \\
\hline $\begin{array}{l}\text { Herbal intake during } \\
\text { pregnancy }\end{array}$ & & & & \\
\hline Takes & 80 & 78 & $3.32(2.14-5.15)$ & $<0.001$ \\
\hline Not take & 66 & 214 & Ref & \\
\hline
\end{tabular}

\section{Maternal factors significantly associated with LBW deliveries}

After multivariable logistic regression analysis, factors found to be significantly associated with delivery of LBW babies included: being unemployment $(A o R=2.52,95 \% \mathrm{Cl} 1.16-5.49, \mathrm{p}=0.020)$, anaemia during pregnancy $(\mathrm{AoR}=3.88,95 \% \mathrm{Cl} 1.90-7.90, \mathrm{p}<0.001)$, having less than two years inter-pregnancy interval $(A \circ R=2.53,95 \% \mathrm{Cl} 1.11-5.73, \mathrm{p}=0.026)$ and cigarette smoking during pregnancy ( $\mathrm{AoR}=4.36,95 \% \mathrm{Cl}$ $1.94-9.80, p<0.001$ ) (Table 5). 
Table 5

Determinants of delivery of LBW babies, WAU, 2020

\begin{tabular}{|c|c|c|c|c|}
\hline Determinants & $\begin{array}{l}\text { Cases }(n= \\
146)\end{array}$ & $\begin{array}{l}\text { Controls }(n= \\
292)\end{array}$ & $\begin{array}{l}\text { AoR } \\
(95 \% \mathrm{Cl})\end{array}$ & $\begin{array}{l}p- \\
\text { value }\end{array}$ \\
\hline \multicolumn{5}{|l|}{ Mother employment } \\
\hline Unemployed & 63 & 50 & $2.52(1.16-5.49)$ & 0.020 \\
\hline Student & 20 & 39 & $2.67(0.93-7.64)$ & 0.066 \\
\hline Employed/self-employed & 63 & 203 & Ref & \\
\hline \multicolumn{5}{|l|}{ Gravidity } \\
\hline Primigravida(1) & 80 & 117 & $1.77(0.80-3.90)$ & 0.152 \\
\hline Multigravida(> 1) & 66 & 175 & Ref & \\
\hline \multicolumn{5}{|l|}{$\begin{array}{l}\text { Mother anaemia status in } \\
\text { pregnancy }\end{array}$} \\
\hline Anaemic $(\mathrm{Hb}<11.0 \mathrm{~g} / \mathrm{dl})$ & 82 & 64 & $3.88(1.90-7.90)$ & $<.001$ \\
\hline Not anaemic $(\mathrm{Hb} \geq 11.0 \mathrm{~g} / \mathrm{dl})$ & 64 & 228 & Ref & \\
\hline \multicolumn{5}{|l|}{ Birth spacing } \\
\hline$<2$ years & 24 & 29 & $2.53(1.11-5.73)$ & 0.026 \\
\hline$\geq 2$ years & 58 & 159 & Ref & \\
\hline \multicolumn{5}{|l|}{ HIV } \\
\hline Positive & 11 & 7 & $\begin{array}{l}4.01(0.92- \\
17.32)\end{array}$ & 0.063 \\
\hline Negative & 135 & 285 & Ref & \\
\hline \multicolumn{5}{|l|}{ Malaria } \\
\hline Positive & 106 & 137 & $1.80(0.88-3.65)$ & 0.103 \\
\hline Negative & 40 & 155 & Ref & \\
\hline \multicolumn{5}{|l|}{ Smoking } \\
\hline Smokes & 52 & 29 & $4.36(1.94-9.80)$ & $<.001$ \\
\hline Does not smoke & 94 & 263 & Ref & \\
\hline
\end{tabular}

$p$-value $<0.05$ is consider statistically significant; AoR (Adjusted odd Ratio) 


\begin{tabular}{|lllll|}
\hline Determinants & $\begin{array}{l}\text { Cases }(n= \\
\text { 146) }\end{array}$ & $\begin{array}{l}\text { Controls }(\mathbf{n}= \\
\mathbf{2 9 2})\end{array}$ & $\begin{array}{l}\text { AoR } \\
(95 \% \mathrm{Cl})\end{array}$ & $\begin{array}{l}\text { p- } \\
\text { value }\end{array}$ \\
\hline Takes & 80 & 78 & $1.92(0.99-3.72)$ & 0.051 \\
\hline Does not take & 66 & 214 & Ref & \\
\hline$p$-value $<0.05$ is consider statistically significant; AoR (Adjusted odd Ratio) & & \\
\hline
\end{tabular}

\section{Discussion}

An unmatched case-control study was conducted among women who delivered in five referral hospitals in the Western Area Urban district of Sierra Leone to determine factors associated with delivery of low birth weight (LBW) babies. This study has identified some socio-demographic, socio-economic, obstetric and lifestyle-related factors that are associated with the delivery of LBW babies in the study area.

Earlier studies from across Africa have reported some association between the delivery of LBW babies and several maternal socio-demographic, socio-economic, obstetric and medical factors [12-14]. In our current study, mothers who were unemployed were found to have a two-fold odds of giving birth to a LBW baby. This is consistent with findings of a population-based study in southern rural Ghana, which established a strong association between birth weight and maternal employment status. Mothers who were unemployed and coming from the poorest households were reported to be more likely to give birth to LBW babies compared to those who were gainfully employed [12]. Unemployment can contribute to poverty, leading to poor maternal nutritional intake [14]. According to Ahmed, et al., maternal undernutrition and inadequate dietary diversity during pregnancy are significant determinants of delivery of low birth weight babies [15].

The odds of delivering a LBW baby as observed in the current study, was greater among mothers who were anaemic during pregnancy $(\mathrm{Hb}<11.0 \mathrm{~g} / \mathrm{dl})$, compared to those who were not, similar to an earlier study also carried out in Ghana [7]. Other studies done in Ethiopia [4], Democratic Republic of Congo [14] and India [16] have also reported similar findings in which anaemia during pregnancy was found to be associated with LBW. Anaemia during pregnancy can limit maternal oxygen uptake, thus reducing oxygen supply to the foetus and this contribute to foetal growth restriction [17].

In this study, babies born within less than two years after another child had higher odds of being of LBW, compared with those born after two or more years. Similar findings have been reported from Ethiopia [18, 19] and India [20]. This might be as a result of insufficient replacement of maternal nutrients used-up during the previous pregnancy and this may lead to reduced foetal development. A similar observation made in South Ethiopia by Mingude, et al. [13] has been explained on the basis that, the short interpregnancy interval is not able to allow the mother enough time to recover from the nutritional burden and stress of the previous pregnancy, resulting in maternal nutrition depletion. The short inter-pregnancy interval is also associated with maternal iron and folic acid depletion [15]. This reduces the ability of the 
mother to support foetal growth and development which in turn increases the possibility of growth restriction and LBW in subsequent pregnancies.

Our study also revealed that mothers who smoke cigarettes have higher odds of giving birth to a LBW baby. In a similar case-control study conducted in China [21], pregnant women who were exposed to even passive smoking had an increased risk of delivering low birth weight babies. Although, our study failed to determine the number of cigarette sticks smoked per day and for how long, other studies have reported that, mothers who are heavy smokers (> 8-10 cigarettes/day) had a higher odds of LBW babies [22]. Cigarette smoking is known to reduce oxygen supply to the foetus in-utero as carbon monoxide and nicotine-associated vasoconstriction reduces uterine and placental blood flow, thereby restricting the growth of the foetus, and hence can contribute to LBW [23]. Other factors including gravidity, maternal illhealth and taking of herbal medicine during pregnancy were not found to be significantly associated with the delivery of LBW babies in the current study after multivariable logistic regression analysis.

Although we employed a case-control study, which we consider an appropriate design for the study, the study is not without limitations. Assessment of some independent variables was liable to recall bias. The measurement we used for maternal smoking may not be the most suitable. Measuring the number of cigarettes sticks smoked by a mother in a day may better show the difference between the cases and controls. These limitations notwithstanding, the study has identified important maternal factors that when addressed, can reduce the incidence of LBW deliveries in Sierra Leone and other developing countries with similar characteristics.

\section{Conclusions}

This study has established that unemployment, anaemia during pregnancy, less than two years of interpregnancy interval, and cigarette smoking during pregnancy are significant factors for the delivery of LBW babies. There is a need for health care managers in WAU district to intensify screening and sensitization on the risk factors of LBW during antenatal sessions to address the identified factors among mothers. Also, the Ministry of Youth Affairs should design welfare programmes that would reduce the high rate of unemployment among women in Sierra Leone.

\section{List Of Abbreviations}




\begin{tabular}{|ll|}
\hline ANC & Antenatal care \\
\hline AOR & Adjusted Odd Ratio \\
\hline BMI & Body mass index \\
\hline COR & Confidence Interval \\
\hline GFELTP & Grude Odd Ratio \\
\hline HIV & Human immunodeficiency virus \\
\hline LBW & Low birth weight \\
\hline NGO & Non-Governmental organization \\
\hline PCMH & Princess Christian Maternity Hospital \\
\hline SD & Standard deviation \\
\hline SLDHS & Sierra Leone demographic health survey \\
\hline SLESRC & Sierra Leone Ethics and Scientific Review Committee \\
\hline SLFETP & Sierra Leone Field Epidemiology Training Programme \\
\hline SPSS & Software package for social sciences \\
\hline WAHO & West African Health Organization \\
\hline WAU & Western Area Urban \\
\hline
\end{tabular}

\section{Declarations}

Ethics approval and consent to participate

This study was approved by the Sierra Leone Ethics and Scientific Review Committee (SLESRC). We obtained permission from the Chief Medical Officer, Ministry of Health and Sanitation (MoHS), and the hospital administration. Signed informed consent was obtained from each mother or her parent or guardian (mothers less than 16 years old) before data collection. The information collected was treated as confidential and codes were used to identify participants.

\section{Consent for publication}

Not applicable.

\section{Availability of data and materials}

All data generated during the current study are included in this published article and its additional file. 


\section{Competing interests}

The authors affirmed that we have no conflicting interests.

\section{Funding}

This thesis work was supported by the University of Ghana and WAHO.

\section{Authors' contributions}

DKK, SS and FA, designed and conducted the study. DKK analyzed the data. DKK, SS, FA, KN, AA_L and EK were responsible for the interpretation of data. DKK drafted the manuscript, which all the authors critically reviewed and approved the final version.

\section{Acknowledgement}

We recognize the technical support given by the staff of the Ghana Field Epidemiology and Laboratory Training Programme (GFELTP).We are also grateful to the West African Health Organization (WAHO) for financial support during the University program of the principal investigator. Our appreciation goes to the management of the Sierra Leone Field Epidemiology Training Programme (SLFETP) for the technical support. Special thanks go to the Ministry of Health and Sanitation, hospital managements, study participants, and research assistants for their diverse contributions.

\section{References}

1. Tshotetsi L, Dzikiti L, Hajison P, Feresu S. Maternal factors contributing to low birth weight deliveries in Tshwane District, South Africa. PLoS ONE. 2019; 14 (3): 1-13

2. Hailu LD, Kebede DL. Determinants of low birth weight among deliveries at a Referral Hospital in Northern Ethiopia. Biomed Res. Int. 2018; 2018. doi: 1155/2018/8169615. Accessed 15 Dec 2020.

3. Muchemi OM, Echoka E, Makokha Factors associated with low birth weight among neonates born at Olkalou District Hospital, Central Region, Kenya. Pan Afr Med J. 2015; 20:108. doi: 10.11604/pamj.2015.20.108.4831. Accessed 23 Dec 2020.

4. Gebrehawerya T, Gebreslasie K, Admasu E, Gebremedhin M. Determinants of Low Birth Weight among Mothers Who Gave Birth in Debremarkos Referral Hospital, Debremarkos Town, East Gojam, Amhara Region, Ethiopia. Neonatal Pediatr. Med. 2018; 04(01):1-6. Accessed 23 Dec 2020.

5. Id LT, Dzikiti L, Hajison P, Id SF. Maternal factors contributing to low birth weight deliveries in Tshwane District, South Africa. 2019; doi.org/10.1371/journal.pone.0213058. Accessed 14 Dec 2020.

6. Demelash H, Motbainor A, Nigatu D, Gashaw K, Melese A. Risk factors for low birth weight in Bale zone hospitals, South-East Ethiopia: a case - control study. BMC Pregnancy Childbirth. doi.org/10.1186/s12884-015-0677-y. Accessed 15 Dec 2020. 
7. Adam Z, Ameme DK, Nortey P, Afari EA, Kenu E. Determinants of low birth weight in neonates born in three hospitals in Brong Ahafo region, Ghana , 2016- an unmatched case-control study. BMC Pregnancy Childbirth. doi.org/10.1186/s12884-019-2315-6. Accessed 15 Dec 2020.

8. Siyoum M, Melese T. Factors associated with low birth weight among babies born at Hawassa University Comprehensive Specialized Hospital, Hawassa, Ethiopia. Ital J Pediatr. 2019; 45: doi.org/10.1186/s13052-019-0637-7. Accessed 15 Dec 2020.

9. Statistics Sierra Leone (SSL) and ICF Maccro, Sierra Leone Demographic and Health Survey 2008; 2009.

10. Statistics Sierra Leone (SSL) and ICF International, Sierra Leone Demographic and Health Survey 2013; 2014.

11. Alemu S, Workicho A, Nigatu M, Bokila T, Wolde T. Determinants of low birth weight in public health facilities, of Kambata Tembaro zone, South Ethiopia. PONS - Med. Cas. 2018; 15: 2. doi: 5937/pomc15-17839.

12. Manyeh AK, Kukula V, Odonkor G, Ekey RA, Adjei A, Narh-Bana S, Akpakli DE, Gyapong Socioeconomic and demographic determinants of birth weight in southern rural Ghana: evidence from Dodowa Health and Demographic Surveillance System. BMC Pregnancy Childbirth. 2016; 6:160. doi: 10.1186/s12884-016-0956-2. Accessed 15 Dec 2020.

13. Mingude AB, Gebretsadik W, Misker D, Woldeamanuel Determinants of low birth weight among live birth newborns delivered at public hospitals in Gamo Gofa Zone, South Ethiopia: Unmatched case control study. SAGE Open Med. 2020; 8: doi: 10.1177/2050312120940544. Accessed 15 Dec 2020.

14. llunga PM, Mukuku O,Mawaw PM, Mutombo AM, Lubala TK, Pongombo MSY, Luhete PK, Wembonyama SO, Kabamba AM, Numbi Risk factors for low birth weight in Lubumbashi, Democratic Republic of the Congo. Med Sante Trop. 2016; 26: 386-390.

15. Ahmed S, Hassen K, Wakayo A health facility based case-control study on determinants of low birth weight in Dassie town, Northeast Ethiopia: the role of nutritional factors. Nutr J. 2018; 17: 103. doi: 10.1186/s12937-018-0409-z. Accessed 15 Dec 2020.

16. Dubey DK, Nath DC. An Epidemiological Model Investigating the Association between Mothers Nutritional Status and Low Birth Weight in India. Health, 2016; 08: 03. doi: 4236/health.2016.83027. Accessed 15 Dec 2020.

17. Sharma SR, Giri S, Timalsina U, Bhandari SS, Basyal B, Wagle K, Shrestha Low birth weight at term and its determinants in a tertiary hospital of nepal:A case-control study. PLoS One, 2015; 10: 4. doi: 10.1371/journal.pone.0123962. Accessed 11 Dec 2020.

18. Hailemichael HT, Debelew GT, Alema HB, Weldu MG, Misgina KH. Determinants of adverse birth outcome in Tigrai region, North Ethiopia: Hospital-based case-control study. BMC Pediatr 2020. org/10.1186/s12887-019-1835-6. Accessed 22 Dec 2020.

19. Endalamaw A, Engeda EH, Ekubagewargies DT, Belay GM, Tefera MA. Low birth weight and its associated factors in Ethiopia: A systematic review and meta-analysis. J. Pediatr. 2018; 44:1. doi: 10.1186/s13052-018-0586-6. Accessed 15 Dec 2020. 
20. Metgud CS,Naik VA, Mallapur Factors Affecting Birth Weight of a Newborn - A Community Based Study in Rural Karnataka, India. PLoS One. 2012; 7: e40040. doi: 10.1371/journal.pone.0040040. Accessed 15 Dec 2020.

21. Xi C, Luo M, Wang T, Wang Y, Wang S, Guo L, Lu C. Association between maternal lifestyle factors and low birth weight in preterm and term births: A case-control study. Reprod. Health, 2020; 17: 1. doi.org/10.1186/s12978-020-00932-9. Accessed 20 Dec 2020.

22. Ko TJ, Tsai LY, Chu LC, Yeh SJ, Leung C, Chen CY, Chou HC,Tsao PN, ChenPC, Hsieh WS. Parental smoking during pregnancy and its association with low birth weight, small for gestational age, and preterm birth offspring: A birth cohort study. Neonatol. 2014; 55: 1.

doi: 10.1016/j.pedneo.2013.05.005. Accessed 15 Nov 2020.

23. Andriani $\mathrm{H}, \mathrm{Kuo} \mathrm{H}-\mathrm{W}$. Adverse effects of parental smoking during pregnancy in urban and rural areas. BMC Pregnancy Childbirth. 2014; 14: 414. doi: 1186/s12884-014-0414-y. Accessed 15 Dec 2020.

\section{Supplementary Files}

This is a list of supplementary files associated with this preprint. Click to download.

- Kargboetaladditionaldatafile1.xls 